\title{
Algorithm optimization for weighted gene co-expression network analysis: accelerating the calculation of Topology Overlap Matrices with OpenMP and SQLite
}

\author{
Min Shuai ${ }^{1}$, Xin Chen ${ }^{1, *}$ \\ ${ }^{1}$ School of Pharmacy, Chengdu University of T.C.M, Chengdu, China, 611137 \\ * To whom correspondence should be addressed.
}

\section{Abstract}

Motivation: Weighted gene co-expression network analysis (WGCNA) is an R package that can search highly related gene modules. The most time-consuming step of the whole analysis is to calculate the Topological Overlap Matrix (TOM) from the Adjacency Matrix in a single thread. This study changes it to multithreading.

Results: This paper uses SQLite for multi-threaded data transfer between $\mathrm{R}$ and $\mathrm{C}++$, uses OpenMP to enable multi-threading and calculates the TOM via an adjacency matrix on a Shared-memory MultiProcessor (SMP) system, where the calculation time decreases as the number of physical CPU cores increases.

Availability and implementation: The source code is available at https://github.com/do-somethings-haha/fast calculate TOM of WGCNA

Contact: chenxin@cdutcm.edu.cn

\section{Introduction}

The weighted gene co-expression network analysis (WGCNA) package of R language can search for highly related gene modules (Langfelder and Horvath, 2008), and then find the potential genes in a certain metabolic pathway, or find the key genes in the gene regulatory network . It can be used for medical diagnosis (Wan et al., 2018) or search for key genes related to a certain disease (Zhi et al., 2018), and can study the mechanism of plant response to environmental stress (Chih-Ta et al., 2019) or the structural genes and genes encoding transcription factors in a certain metabolic pathway (Lu et al., 2019). 
Changing the single-threaded algorithm to the multi-threaded algorithm is a major trend in algorithm development. In recent years, the increase in CPU single-core frequency has been limited, but the number of CPU cores has been increasing, and tools for parallel computing have gradually developed, such as OpenMP, CUDA, and MPI(Yang et al., 2010). If single-threaded algorithms can be changed to multi-threaded algorithms, it will be extremely improve the calculation speed. For example, the single-threaded algorithm of sequence comparison has been changed to the multi-threaded algorithm (M et al., 2017), and the algorithm of protein sequence search has been changed to the multi-threaded algorithm (Zhang et al., 2016).

The TOMsimilarity function of the WGCNA package in R language that calculates the Topological Overlap Matrix (TOM) from the Adjacency Matrix is a single-threaded function, and the calculation takes too long, which hinders the use of this method in big data analysis.

This paper changes the algorithm of calculating TOM to a multi-threaded algorithm, which uses SQLite (https://www.sqlite.org/index.html) to realize multi-threaded high-speed data transmission between $\mathrm{R}$ and $\mathrm{C}++$ languages, and uses OpenMP (https://www.openmp.org/) to realize multi-threaded calculation of TOM in C++ language. On a Shared-memory MultiProcessor (SMP) system, the calculation time decreases as the number of CPU cores used increases.

\section{Methods}

\subsection{Data source}

The test data used in this paper was downloaded from GSE61357 (EWipf et al., 2014) of Gene Expression Omnibus (GEO), which used gene chips to measure the expression of 30,677 genes. This paper only uses the expression matrix data of 19 samples whose sampling sites are root (GSM1502821 39).

\subsection{Calculate Adjacency Matrix with WGCNA}

All data of 19 samples from the test data are used in this paper, and the expression matrix is transposed so that the row names are sample names, the column names are the gene IDs. The soft threshold is set to 20. The Adjacency Matrix is calculated by the adjacency function of WGCNA package in $\mathrm{R}$ language.

\subsection{Transfer Adjacency Matrix to $C_{++}$from $\mathbf{R}$}

The 7G Adjacency Matrix is split into many data frames with a maximum column number of 2000, and these data frames are placed in a list. The foreach package in $\mathrm{R}$ language is used to start multiple processes, and each process obtains one data frame from the aforementioned list, while each process creates a connection to the SQLite database and creates a database file with one table. Every process writes a data frame to the corresponding table in the database file. 
$\mathrm{C}++$ language uses OpenMP to start multiple threads. Each thread connects to the corresponding database file, and reads the table in it. Finally, the Adjacency Matrix is stored in a two-dimensional array in $\mathrm{C}++$ language.

\subsection{Calculate TOM using $\mathrm{C}++$}

\subsection{1 convert the formulas}

According to the introduction of TOM in the WGCNA package, the formulas for calculating TOM from the Adjacency Matrix are as follows.

\subsubsection{TOMType $=$ "unsigned", TOMDenom = "min"}

If the parameters of TOMsimilarity function are set to TOMType = "unsigned", and TOMDenom = "min", then use the following formula

$$
\begin{aligned}
& T_{i j}=\frac{u \neq i, j}{\min \left(k_{i}, k_{j}\right)+1-a_{i j}} \\
& k_{i}=a_{u \neq i} a_{i u}
\end{aligned}
$$

where TOM is the the Topological Overlap Matrix, $a$ is the Adjacency Matrix, $i$ is the row number of the Adjacency Matrix and TOM, $j$ is the column number of the Adjacency Matrix and TOM. $u$ increases from 1 to the maximum row number, also the maximum column number.

Because the Adjacency Matrix is a symmetric matrix, and the intermediate diagonals are all 1 , we can deduce that

$$
\begin{aligned}
& a_{u j}=a_{j u} \\
& a_{i i}=a_{j j}=1 \\
& i_{\max }=j_{\max }
\end{aligned}
$$

In combination with (2), (3) and (4), the formula (1) can be transformed into

$$
\begin{aligned}
\text { TOM }_{i j} & =\frac{\sum a_{i u} a_{j u}-a_{i i} a_{i j}-a_{i j} a_{j j}+a_{i j}}{\min \left(\sum a_{i u}-a_{i i}, \sum a_{j u}-a_{j j}\right)+1-a_{i j}} \\
& =\frac{\sum a_{i u} a_{j u}-a_{i j(}\left(a_{i i}+a_{j j)}+a_{i j}\right.}{\min \left(\sum a_{i u}, \sum a_{j u}\right)-1+1-a_{i j}} \\
& =\frac{\sum a_{i u} a_{j u}-a_{i j}\left(a_{i i}+a_{j j)}+a_{i j}\right.}{\min \left(\sum a_{i u}, \sum a_{j u}\right)-1+1-a_{i j}} \\
& =\frac{\sum a_{i u} a_{j u}-a_{i j}}{\min \left(\sum a_{i u}, \sum a_{j u}\right)-a_{i j}}
\end{aligned}
$$




\subsubsection{TOMType = "unsigned", TOMDenom = "mean"}

If the parameters of TOMsimilarity function are set to TOMType = "unsigned", and TOMDenom = "mean", then use the following formula

$$
\operatorname{mcdTOM}_{i j}=\frac{{ }_{u \neq i,} a_{i u} a_{u j}+a_{i j}}{\frac{1}{2}\left(k_{i}+k_{j}\right)+1-a_{i j}}
$$

where $m c d T O M$ is a more conservative modification of TOM.

In combination with (2), (3) and (4), the formula (7) can be transformed into

$$
\begin{aligned}
\operatorname{mcdTOM}_{i j} & =\frac{\sum a_{i u} a_{j u}-a_{i i} a_{i j}-a_{i j} a_{j j}+a_{i j}}{\frac{1}{2}\left(\sum a_{i u}-a_{i i}+\sum a_{j u}-a_{j j}\right)+1-a_{i j}} \\
& =\frac{\sum a_{i u} a_{j u}-a_{i j}\left(a_{i i}+a_{j j}+a_{i j}\right.}{\frac{1}{2}\left(\sum a_{i u}+\sum a_{j u}\right)-1+1-a_{i j}} \\
& =\frac{\sum a_{i u} a_{j u}-a_{i j}}{\frac{1}{2}\left(\sum a_{i u}+\sum a_{j u}\right)-a_{i j}}
\end{aligned}
$$

\subsubsection{Calculate the intermediate values}

Calculating the $\sum a_{i u} a_{j u}$ of the numerator in formula (6) or (8). According to the formula (6) or (8), the total number of intermediate values in the numerator is equal to the combination number: $\frac{i \text { max } !}{2 !(i \max -2) !}$. C++ language uses multiple threads to calculate these values and store them in a one-dimensional array.

Calculating $\sum a_{i u}$ and $\sum a_{j u}$ of the numerator in formula (6) or (8). C++ language uses multiple threads to calculate these values and then stores the values in a one-dimensional array.

\subsubsection{Calculate the final results}

This paper uses the intermediate values to calculate each value of TOM according to (6) or (8).

\subsection{Transfer TOM to $\mathbf{R}$ from $\mathbf{C}++$}

$\mathrm{C}++$ language uses OpenMP to start multiple threads, and each thread connects to a database file. The TOM is split into multiple two-dimensional arrays with a maximum 
number of 2000. Every two-dimensional array is written into one table of the corresponding database file.

$\mathrm{R}$ language uses the foreach package to create multiple processes, and each process reads one of the aforementioned tables. Then, using the cbind function to bind the results of each process into a matrix named TOM.

\section{Results}

The results of the optimised multi-threaded algorithm are identical to the single-threaded results of the WGCNA package's TOMsimilarity function, and they differ only in the last significant figures of the double-precision floating-point number due to the truncation error.

This paper tests the algorithms in shared memory multi-processor (SMP) system(CPU: Dual Intel Xeon Gold 5220 Processors, 36 cores in total. Memory: $8 \times 32$ G, ECC, DDR4, 2666MHz, Operating system: Ubuntu 20.04). The WGCNA package's TOMsimilarity function takes 180 minutes with a peak memory usage of about 10G. This paper's algorithm takes 13 minutes if 72 threads are used with a peak memory usage of about $40 \mathrm{G}$. The parallel acceleration ratio is 13.8 , and the average acceleration ratio is 0.38 . The relationship between the number of threads and the total time consumption is plotted in Figure 1.

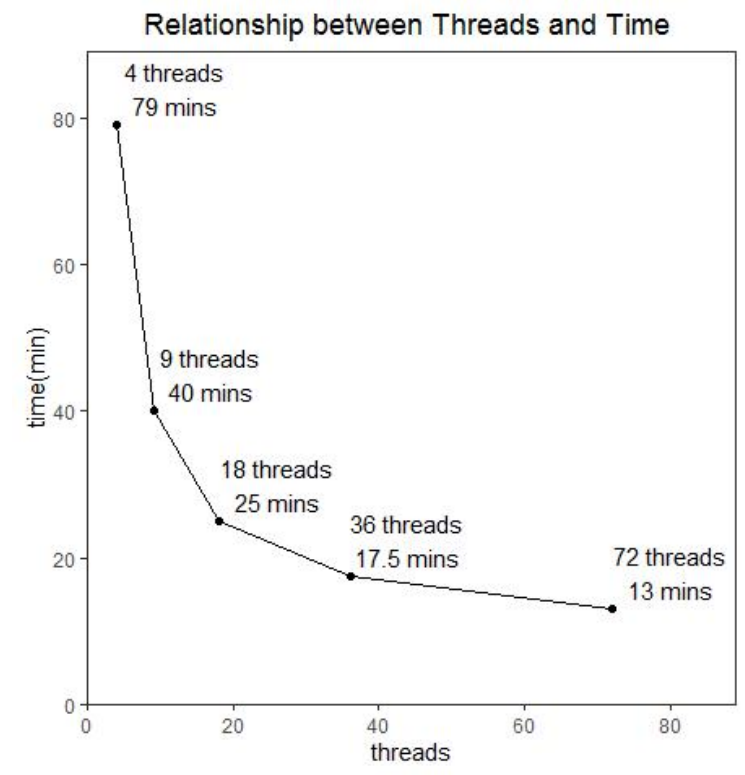

Fig. 1. The relationship between the number of threads and the total time consumption for the optimized algorithm in this paper (CPU cores:36, total gene number: 30677). The WGCNA package's single-threaded TOMsimilarity function takes 180 minutes.

\section{Conclusions}

There are a variety of clustering algorithms to search co-expressed genes, such as means of double clustering (Qinghui et al. , 2012), k-means, approximate kernel k-means, 
c-means, and hierarchical clustering (Uygun et al., 2016). There are also many methods for constructing gene regulatory networks, however, the WGCNA package in R language is one of the best methods (Ruyssinck et al., 2016). Since the release of the WGCNA package, there are lots of improvements, such as optimizing the degree of matching between gene modules and traits (David et al., 2019).

If the algorithm of this paper is used to calculate the Adjacency Matrix with more than 10,000 genes, it is recommended to run on Linux systems, large-capacity ECC memory banks, server CPUs such as Intel Xeon series or AMD EPYC series, and good radiators.

The algorithm optimized in this paper greatly improves the calculation speed of the most time-consuming step in the WGCNA package. Thereby, the optimized algorithm can significantly improve the speed of the entire weighted gene co-expression network analysis, and it will enable the WGCNA package to be used more widely in the future, such as analyzing data from single cell sequencing (Luo et al., 2015).

\section{References}

\section{References:}

Chih-Ta, L. et al. (2019) Weighted Gene Co-expression Network Analysis (WGCNA) Reveals the Hub Role of Protein Ubiquitination in the Acquisition of Desiccation Tolerance in Boea hygrometrica., Plant \& cell physiology, 60.

David, T. et al. (2019) A Genetic Algorithm to Optimize Weighted Gene Co-Expression Network Analysis., Journal of computational biology : a journal of computational molecular cell biology, 26.

EWipf, D. et al. (2014) Transcriptional responses of Medicago truncatula upon sulfur deficiency stress and arbuscular mycorrhizal symbios, FRONT PLANT SCI, 5.

Langfelder, P. and Horvath, S. (2008) WGCNA: an R package for weighted correlation network analysis, BMC BIOINFORMATICS, 9.

Lu, C. et al. (2019) Comparative transcriptomics and weighted gene co-expression correlation network analysis (WGCNA) reveal potential regulation mechanism of carotenoid accumulation in Chrysanthemum $\times$ morifolium, PLANT PHYSIOL BIOCH, 142.

Luo, Y. et al. (2015) Single-Cell Transcriptome Analyses Reveal Signals to Activate Dormant Neural Stem Cells, CELL, 161.

M, A.J. et al. (2017) PASTASpark: multiple sequence alignment meets Big Data., Bioinformatics (Oxford, England), 33.

Qinghui, G. et al. (2012) Biclustering of linear patterns in gene expression data., Journal of computational biology : a journal of computational molecular cell biology, 19.

Ruyssinck, J. et al. (2016) Netter: re-ranking gene network inference predictions using structural network properties, BMC BIOINFORMATICS, 17.

Uygun, S. et al. (2016) Utility and Limitations of Using Gene Expression Data to Identify Functional Associations, PLOS COMPUT BIOL, 12. 
Wan, Q. et al. (2018) Co-expression modules construction by WGCNA and identify potential prognostic markers of uveal melanoma, EXP EYE RES, 166.

Yang, C. et al. (2010) Hybrid CUDA, OpenMP, and MPI parallel programming on multicore GPU clusters, COMPUT PHYS COMMUN, 182.

Zhang, J. et al. (2016) muBLASTP: database-indexed protein sequence search on multicore CPUs, BMC BIOINFORMATICS, 17.

Zhi, Z. et al. (2018) Weighted Gene Correlation Network Analysis (WGCNA) Detected Loss of MAGI2 Promotes Chronic Kidney Disease (CKD) by Podocyte Damage., Cellular physiology and biochemistry : international journal of experimental cellular physiology, biochemistry, and pharmacology, 51. 